\title{
New Equilibrium Models of Drug-Receptor Interactions Derived from Target-Mediated Drug Disposition
}

\author{
Lambertus A. Peletier ${ }^{1,3}$ and Johan Gabrielsson ${ }^{2}$
}

Received 5 February 2018; accepted 23 March 2018; published online 14 May 2018

\begin{abstract}
In vivo analyses of pharmacological data are traditionally based on a closed system approach not incorporating turnover of target and ligand-target kinetics, but mainly focussing on ligand-target binding properties. This study incorporates information about target and ligand-target kinetics parallel to binding. In a previous paper, steady-state relationships between target- and ligand-target complex versus ligand exposure were derived and a new expression of in vivo potency was derived for a circulating target. This communication is extending the equilibrium relationships and in vivo potency expression for $(i)$ two separate targets competing for one ligand, (ii) two different ligands competing for a single target and (iii) a single ligand-target interaction located in tissue. The derived expressions of the in vivo potencies will be useful both in drug-related discovery projects and mechanistic studies. The equilibrium states of two targets and one ligand may have implications in safety assessment, whilst the equilibrium states of two competing ligands for one target may cast light on when pharmacodynamic drug-drug interactions are important. The proposed equilibrium expressions for a peripherally located target may also be useful for small molecule interactions with extravascularly located targets. Including target turnover, ligand-target complex kinetics and binding properties in expressions of potency and efficacy will improve our understanding of within and between-individual (and across species) variability. The new expressions of potencies highlight the fact that the level of drug-induced target suppression is very much governed by target turnover properties rather than by the target expression level as such.
\end{abstract}

KEY WORDS: drug disposition; drug-target interaction; multi-drug target binding; multi-target drug disposition.

\section{INTRODUCTION}

\section{Background}

In this paper, we continue our study of in vivo potency of drug-target kinetics begun in Gabrielsson, Peletier et al. and Hjorth et al. $(1,2)$ in the framework of Target-Mediated Drug Disposition (TMDD), an ubiquitous process in the action of drugs that has been extensively studied ever since the

\footnotetext{
${ }^{1}$ Mathematical Institute, Leiden University, PB 9512, 2300 RA, Leiden, The Netherlands.

${ }^{2}$ Department of Biomedical Sciences and Veterinary Public Health, Division of Pharmacology and Toxicology, Swedish University of Agricultural Sciences, Box 7028, 750 07, Uppsala, Sweden.

${ }^{3}$ To whom correspondence should be addressed. (e-mail: peletier@math.leidenuniv.nl)
}

pioneering papers of Wagner (3), Sugiyama et al. (4) and Levy (5). We also refer to the seminal papers by Michaelis and Menten (6), Mager and Jusko (7), Mager and Krzyzansky (8), Gibiansky et al. (9) and Peletier and Gabrielsson (10). In Fig. 1, we show schematically the basic TMDD model: Ligand is supplied to the central compartment where it binds a receptor (the target) resulting in a ligand-receptor complex, which internalises to produce a pharmacologial response. In addition, ligand is cleared from the central compartment and exchanged with a peripheral compartment. Target is synthesised by a zeroth order process and degrades by a first-order process.

In this paper, we extend the results for this TMDD model obtained in (1) to three generalisations of the TMDD model in which (i) the drug can bind two receptors (cf. 11), (ii) two drugs can bind one receptor (cf. 12) and (iii) the dug is supplied to the central compartment, but the receptor is located in the peripheral compartment (cf. 13). 


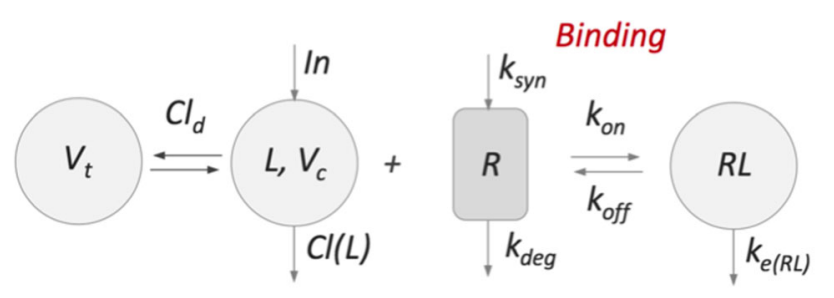

Non-specific Target Internalisation elimination turnover

Fig. 1. Schematic description of the model for Target Mediated Drug Disposition involving ligand in the central compartment $\left(L_{\mathrm{c}}\right)$ and in the peripheral compartment $\left(L_{\mathrm{p}}\right)$ binding a receptor $(R)$ (the target), yielding ligand-target complexes $(R L)$

Mathematically, the basic TMDD model, depicted in Fig. 1, can be formulated as a set of four differential equations, one for each compartment.

$$
\left\{\begin{array}{l}
\frac{d L_{c}}{d t}=\frac{I n}{V_{c}}-k_{\mathrm{on}} L_{c} \cdot R+k_{\mathrm{off}} R L+\frac{C l_{d}}{V_{c}}\left(L_{p}-L_{c}\right)-\frac{C l_{(L)}}{V_{c}} L_{c} \\
\frac{d L_{p}}{d t}=\frac{C l_{d}}{V_{p}}\left(L_{c}-L_{p}\right) \\
\frac{d R}{d t}=k_{\mathrm{syn}}-k_{\mathrm{deg}} R-k_{\mathrm{on}} L_{c} \cdot R+k_{\mathrm{off}} R L \\
\frac{d R L}{d t}=k_{\mathrm{on}} L_{c} \cdot R-\left(k_{\mathrm{off}}+k_{e(R L)}\right) R L
\end{array}\right.
$$

Here, $L_{c}$ and $L_{p}$ denote the concentrations of ligand (or drug) in, respectively, the central and the peripheral compartment with volumes $V_{c}$ and $V_{p}$. Concentrations of target and target-ligand complex in the central compartment are denoted by $R$ and $R L$. Drug infusion takes place into the central compartment, with constant rate In where it binds to the target with rates $k_{\text {on }}$ and $k_{\text {off }}$ Ligand is removed through non-specific clearance $C l_{(L)}$ and exchanged with the peripheral compartment through inter-compartmental distribution $C l_{d}$. By internalisation, ligand-target complex leaves the system according to a first-order process with a rate constant $k_{e(R L)}$. Finally, target synthesis and degradation are modelled by, respectively, zeroth- and first-order turnover with rates $k_{\text {syn }}$ and $k_{\text {deg. }}$.

We recall the analysis presented in (1) for the onecompartment TMDD-model shown in Fig. 1. There, relations between steady-state concentrations of target $R$, ligand $L$ and complex $R L$ were derived, and a new expression of the in vivo potency, denoted by $L_{50}$, was established, particularly suited for Open Systems. Whereas the classical definition of potency is primarily based on the binding constants (cf. Black and Leff (14), Kenakin (15,16), Neubig et al. (17)) and target expression, in the definition of, in vivo potency drug and target kinetics, such as the degradation rate $k_{\mathrm{deg}}$, are also incorporated. These concepts were further discussed from an open and closed system perspective in (2).

In this paper, we present three generalisations of the classical TMDD model: (i) a single ligand that can bind two receptors $R_{1}$ and $R_{2}$, (ii) two ligands, $L_{1}$ and $L_{2}$, that compete for a single receptor and (iii) a ligand that is supplied to the central compartment and distributed to the peripheral compartment where the target is located.

\section{Steady States}

In (1), it has been established how for the model shown in Fig. 1, the steady-state values of ligand $(L)$, receptor $(R)$ and ligand-receptor complex $(R L)$, in the central compartment, are related to one another:

$R L=R^{*} \cdot \frac{L}{L+L_{50}} \quad$ and $\quad R=R_{0} \cdot \frac{L_{50}}{L+L_{50}}$

where the baseline $R_{0}$, the maximal impact $R^{*}$ and the in vivo potency $E C_{50}$ (denoted by $L_{50}$ ) are given by

$R_{0}=\frac{k_{s y n}}{k_{d e g}}, \quad R^{*}=\frac{k_{s y n}}{k_{e(R L)}} \quad$ and $\quad L_{50}=\frac{k_{d e g}}{k_{e(R L)}} \cdot K_{m}$

and $K_{m}=\left(k_{\text {off }}+k_{e(R L)}\right) / k_{\text {on }}$ is called the Michaelis-Menten constant. Here, it is implicitly assumed that the constant rate infusion, In, is fixed at the appropriate value. In (1), the required infusion rate is also computed.

The definition of the in vivo potency, $L_{50}$, expresses both the impact of rate processes of the target $\left(k_{\mathrm{deg}}, k_{e(R L)}\right)$ and those of the binding dynamics $\left(k_{\text {off }}, k_{\text {on }}\right)$, on the drug concentration $(L)$ required to achieve the desired efficacy.

The resemblance of Eq. (2) with the Hill equation (below) is striking.

$E=E_{0} \pm E_{\max } \frac{C^{n_{H}}}{E C_{50}^{n_{H}}+C^{n_{H}}}$.

The Hill equation is often used in vivo and also contains a baseline parameter $E_{0}$ in addition to the maximum drug induced effect $E_{\max }$ and the potency $E C_{50}$. Equation (2) has intrinsically the baseline in terms of $R_{0}$. The $E_{\max }$ parameter is equivalent to $\left|R L_{\max }-R_{0}\right|$, and the potency parameter $E C_{50}$ is expressed in Eq. (3) as $L_{50}$.

The exponent $n_{H}$ of the Hill equation is interpreted as a fudge factor allowing the steepness of the Hill equation at the $E C_{50}$ value to vary. In our experience $n_{H}$ is not necessarily an integer and varies typically within the range of $1-3$. We have observed with high and variable plasma protein binding that $n_{H}$ will change depending on whether unbound or total plasma concentration (respectively $C_{u}$ and $C_{\text {tot }}$ ) is used as drivers of the pharmacological effect.

Remark. It is interesting to note that Eq. (3) yields the following relation between the baseline target concentration, $R_{0}$, the maximal ligand-target concentration, $R^{*}$, and the in vivo potency $L_{50}$ :

$L_{50} \cdot R_{0}=K_{m} \cdot R^{*}$

This means that if the baseline of target, the maximum ligandtarget concentration and $K_{m}$ are obtained experimentally, then the in vivo potency $L_{50}$ can be predicted. Thus, $K_{m}$ can be located either to the right or to the left of the in vivo potency, depending on the relative magnitude of $R_{0}$ and $R^{*}$.

In Fig. 2, we show graphs for $R L$ and $R$ versus $L$ for two parameter sets, one taken from Peletier and Gabrielsson (10) 
(left) and one from Cao and Jusko (18) (right) (cf. Appendix 2; Tables I and II).

The values of $R_{0}, R^{*}$ and $L_{50}$ that appear in Eq. (2) are for these two references given by

$$
\begin{array}{ll}
R_{0}=12, \quad R^{*}=36 \quad L_{50}=0.13 & \text { Peletier and Gabrielsson }[10] \\
R_{0}=10 \quad R^{*}=3.3 \quad L_{50}=0.10 & \text { Cao and Jusko }[18]
\end{array}
$$

Thus, remembering that initially, $R=R_{0}$ and $R L=0$, it is evident that over time, the system settles into a steady state, in (10) where total target concentration exceeds $R_{0}$ and in (18) where target concentration is less than target baseline.

It is interesting to note that despite similar in vivo potency's ( $L_{50}$ 's) of Cao and Jusko and Peletier and Gabrielsson, the target-to-complex ratios differ by one order of magnitude due to the comparable difference in $k_{e(R L)}$.

The proposed framework with a dynamic target protein may also be applicable to enzymatic reactions which may enhance the in vitro/in vivo extrapolation of metabolic data (cf Pang et al. 19,20).

\section{Discussion and Conclusions}

Eqs. (2) and (3) summarise what is needed to apply and explain target $R$, ligand-target $R L$ and ligand $L$ interactions when both ligand and target belongs to the central (plasma) compartment. Equation (3) clearly demonstrates that in vivo potency, a central parameter in pharmacology, is a conglomerate of target turnover, complex kinetics and ligand-target binding properties.

In the following three sections, we discuss generalisations of the basic TMDD model discussed in "INTRODUCTION" and derive generalisations of the functions $R L=f(L)$ and $R=$ $g(L)$ applicable to these models.

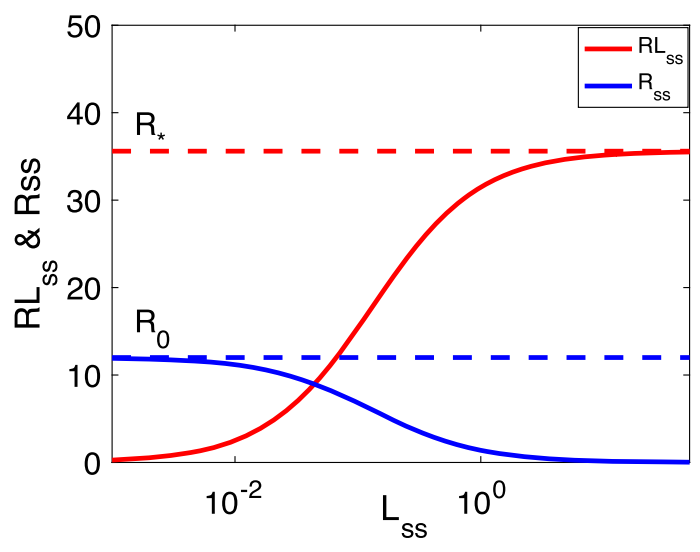

TWO DIFFERENT RECEPTORS COMPETE FOR ONE LIGAND

\section{Background}

When one ligand, $L$, can bind two receptors, $R_{1}$ and $R_{2}$, two complexes are formed and internalised to form two different ligand-receptor complexes $R_{1} L$ and $R_{2} L$; it is of great value to determine their relative impact on the pharmacological response, and it is important to determine how the responses of these two complexes are related. For instance, when one receptor mediates a beneficial effect of a drug and the other one mediates an adverse effect, one wishes to know the relative impact of the latter target and whether the two potencies $E C_{50 ; 1}$ and $E C_{50 ; 2}$ are sufficiently well separated so that a dose can be selected with minimally adverse effect. Figure 3 gives a schematic description of the model.

Mathematically, the model shown in Fig. 3 can be described by the following system of ordinary differential equations:

$$
\left\{\begin{array}{l}
\frac{d L}{d t}=k_{\mathrm{infus}}-k_{e(L)} L-k_{\mathrm{on} ; 1} L \cdot R_{1}+k_{\mathrm{off} ; 1} R_{1} L \\
\quad-k_{\mathrm{on} ; 2} L \cdot R_{2}+k_{\mathrm{off} ; 2} R_{2} L \\
\frac{d R_{1}}{d t}=k_{\mathrm{syn} ; 1}-k_{\mathrm{deg} ; 1} R_{1}-k_{\mathrm{on} ; 1} L \cdot R_{1}+k_{\mathrm{off} ; 1} R_{1} L \\
\frac{d R_{1} L}{d t}=k_{\mathrm{on} ; 1} L \cdot R_{1}-\left(k_{\mathrm{off} ; 1}+k_{e\left(R_{1} L\right)}\right) R_{1} L \\
\frac{d R_{2}}{d t}=k_{\mathrm{syn} ; 2}-k_{\mathrm{deg} ; 2} R_{2}-k_{\mathrm{on} ; 2} L \cdot R_{2}+k_{\mathrm{off} ; 2} R_{2} L \\
\frac{d R_{2} L}{d t}=k_{\mathrm{on} ; 2} L \cdot R_{2}-\left(k_{\mathrm{off} ; 2}+k_{e\left(R_{2} L\right)}\right) R_{2} L
\end{array}\right.
$$

in which the parameters are defined as in the system (1) and

$k_{\text {infus }}=\frac{I n}{V_{c}} \quad$ and $\quad k_{e(L)}=\frac{C l_{(L)}}{V_{c}}$

For a related model, with a corresponding system of

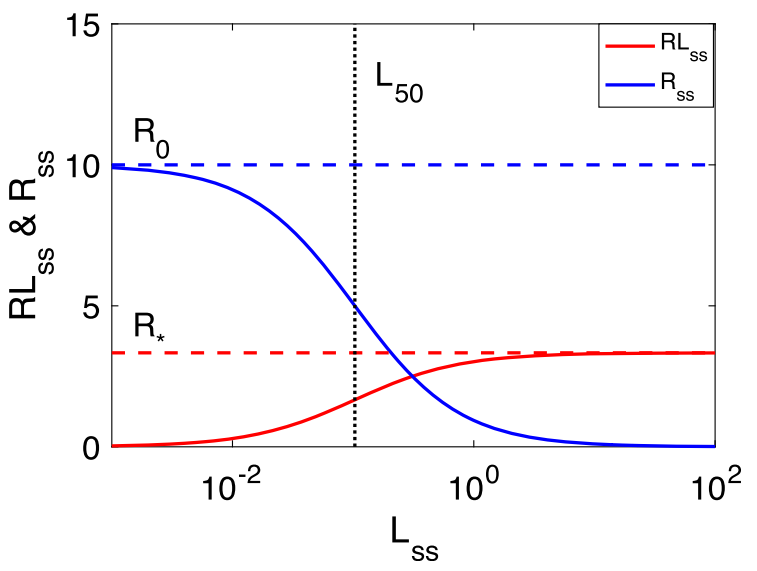

Fig. 2. $R L_{s s}$ and $R_{s s}$ versus $L_{s s}$ for the parameter values of Peletier and Gabrielsson (10) (left) and Cao and Jusko (18) (right). The parameter values are given in Tables I and II in Appendix 2 


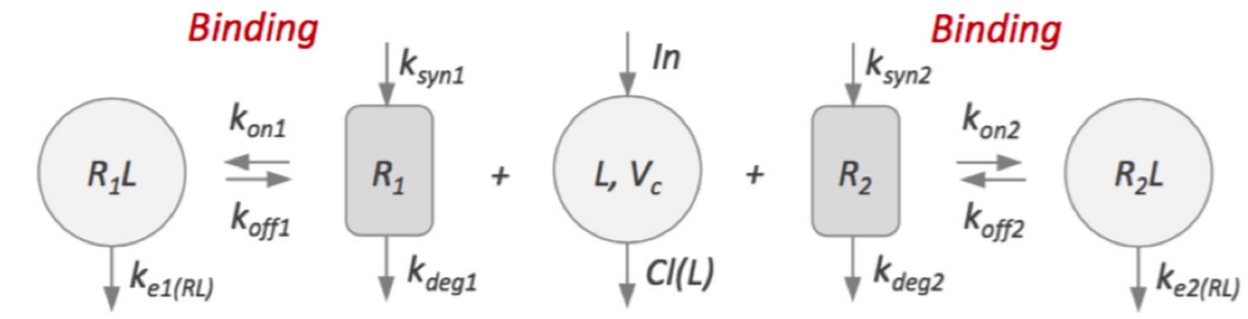

\section{Internalisation Target Non-specific Target \\ turnover elimination turnover \\ Internalisation}

Fig. 3. Schematic description of a model for the one-compartment two-target system in which ligand binds with two receptors $R_{1}$ and $R_{2}$, each forming a complex denoted by, respectively, $R_{1} L$ and $R_{2} L$. The definition of parameters is the same as in Fig. 1

equations, we refer to (11). By adding the equations for free receptors $R_{1}$ and $R_{2}$ to the equations for the associated bound receptors $R_{1} L$ and $R_{2} L$, we obtain two balance equations for, respectively, $R_{1}$ and $R_{2}$ :

$$
\left\{\begin{array}{l}
\frac{d}{d t}\left(R_{1}+R_{1} L\right)=k_{\mathrm{syn} ; 1}-k_{\mathrm{deg} ; 1} R_{1}-k_{e\left(R_{1} L\right)} R_{1} L \\
\frac{d}{d t}\left(R_{2}+R_{2} L\right)=k_{\mathrm{syn} ; 2}-k_{\mathrm{deg} ; 2} R_{2}-k_{e\left(R_{2} L\right)} R_{2} L
\end{array}\right.
$$

For ligand, free or bound to one of the two receptors, we obtain the balance equation:

$\frac{d}{d t}\left(L+R_{1} L+R_{2} L\right)=k_{\mathrm{inf}}-k_{e(L)} L-k_{e\left(R_{1} L\right)} R_{1} L-k_{e\left(R_{2} L\right)} R_{2} L(9)$

\section{Steady States}

As in the case of a single target, it is possible to obtain expressions for the concentrations of ligand-target complex and free target, i.e. for $R_{i} L$ and $R_{i}(i=1,2)$ in terms of the ligand concentration $L$.

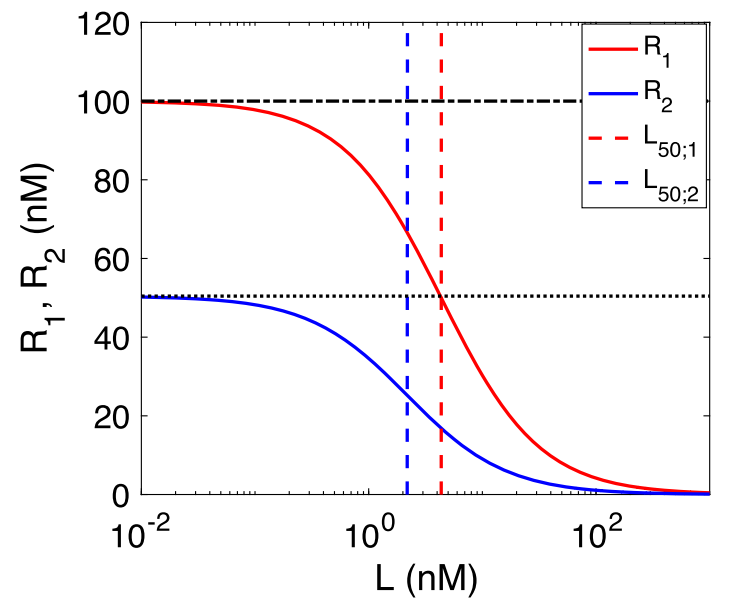

Following the steps taken in Gabrielsson and Peletier (1), it is possible to show that for the receptors individually, the expressions such as shown in (2) hold

$R_{i}=R_{0 . i} \cdot \frac{L_{50 ; i}}{L+L_{50 ; i}} \quad$ and $\quad R_{i} L=R_{i}^{*} \cdot \frac{L}{L+L_{50 ; i}}$

for $i=1$ and $i=2$.

The baseline receptor concentrations $R_{0, i}$, the maximum values $R_{i}^{*}$ of the receptor-ligand complexes and the in vivo potencies $L_{50 ; i}$ are given by

$R_{0, i}=\frac{k_{s y n ; i}}{k_{\text {deg } ; i}}, \quad R_{i}^{*}=\frac{k_{s y n ; i}}{k_{e\left(R_{i} L\right)}}, \quad L_{50 ; i}=\frac{k_{\text {deg } ; i}}{k_{e\left(R_{i} L\right)}} \cdot K_{m ; i}$

for $i=1$ and $i=2$.

where $K_{m ; i}=\left(k_{\mathrm{off} ; i}+k_{e\left(R_{i} L\right)}\right) / k_{\mathrm{on} ; i}$. Details of the derivations of the formulas above are presented in Appendix 1.1.

Figure 4 shows the target suppression and complex formation of the two targets versus ligand concentration at equilibrium together with their respective $L_{50}$ values. These graphs are useful in discriminating between two targets and deciding which target contributes most to complex formation at different ligand concentrations. The left figure shows the

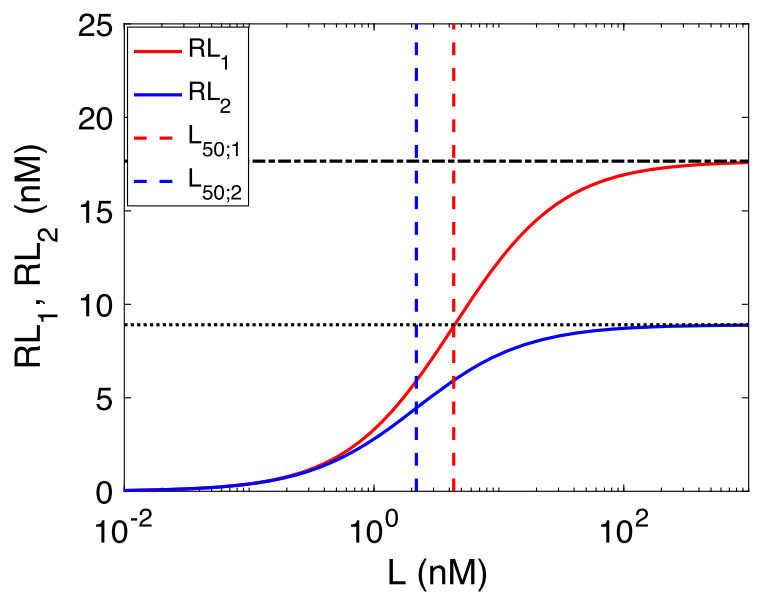

Fig. 4. Target suppression (left) and ligand-target complex (right) versus ligand concentration for two receptors $\boldsymbol{R}_{\mathbf{1}}$ and $\boldsymbol{R}_{\mathbf{2}}$. The parameter values for the two receptors are given in Table II in Appendix 2. The dashed lines indicate the corresponding values for $\boldsymbol{L}_{\mathbf{5 0}}: \boldsymbol{L}_{\mathbf{5 0 ; 1}}=\mathbf{4 . 3 4} \mathrm{nM}$ and $\boldsymbol{L}_{\mathbf{5 0 ; 2}}=\mathbf{2 . 1 8} \mathrm{nM}$ 
two target suppression curves $R_{1}$ and $R_{2}$ versus ligand and the right figure the two ligand-target complexes $R_{1} L$ and $R_{2} L$ versus ligand $L$. The parameter values are chosen fictitiously in order to clearly highlight the differences (Table III, Appendix 2).

The model shown in Fig. 3 has been used as a two-state model to fit the data for the total free target concentration that were given in Gabrielsson and Weiner (PD2) p. 729 (21). The total free target concentration, i.e. $R_{1}+R_{2}$, can be computed from Eq. (10) and (11) and is seen to be

$$
R_{\text {free;tot }}=R_{1}+R_{2}=R_{0.1} \cdot \frac{L_{50 ; 1}}{L+L_{50 ; 1}}+R_{0.2} \cdot \frac{L_{50 ; 2}}{L+L_{50 ; 2}}
$$

Evidently, in the absence of ligand, $R_{\text {free; tot }}=R_{0.1}+R_{0.2}$, while $R_{\text {free; tot }} \rightarrow 0$ as $L \rightarrow \infty$.

In Fig. 5, we see how the model is fitted to data obtained from an experiment involving four total target concentrations $\left(R_{\text {tot }}=8050,6510,3540\right.$ and $\left.1590 \mathrm{nM}\right)$. As the ligand concentration increases, the first receptor kicks in at the lowest in vivo potency $(0.025 \mathrm{nM})$, taking the free receptor concentration down to a lower intermediate plateau. Then, at the higher in vivo potency $(37 \mathrm{nM})$, the free receptor concentration drops further and eventually converges to zero.

Remark. The parameters $k_{\mathrm{deg}}, k_{e(R L)}, k_{\mathrm{on}}$ and $k_{\mathrm{off}}$ are not given here since only equilibrium data from the experiments were available. Due to parameter unidentifiability, the model was parametrised with potencies $L_{50 ; 1}$ and $L_{50 ; 2}$ as parameters and not functions of their original determinants. One may also need other sources of information to fully appreciate the actual values of $k_{\mathrm{deg}}, k_{e(R L)}, k_{\text {on }}$ and $k_{\text {off }}$. In vitro binding experiments may yield $k_{\text {on }}$ and $k_{\text {off. In vivo }}$ time courses of circulating free ligand, target and ligandtarget are necessary in order to estimate $k_{e(R L)}$. Information about the $k_{\text {deg }}$ parameter may be found in the literature for commonly studied targets.

\section{Discussion and Conclusion}

Here, Eq. (10), (11) and (12) summarise what is needed to apply and explain target $R_{i}$, ligand-target $R_{i} L$ and ligand $L$ interactions when ligand and both targets belong to the central (plasma) compartment. Equation (11) demonstrates again the complexity of in vivo potencies $L_{50 ; i}$ involving both turnover of the two targets, complex kinetics and ligandtarget binding properties.

The explicit expressions for the ligand-receptor complexes $R_{i} L$, the free receptor concentrations $R_{i}$ and the in vivo potencies $L_{50 ; i}$ (cf. (12)), together with Figs. 4 and 5 , provide valuable tools when assessing the individual contribution of each target and specifically the impact of target turnover and internalisation.

\section{TWO DIFFERENT LIGANDS COMPETING FOR ONE RECEPTOR}

\section{Background}

A common situation, for instance in combination therapy, is that not one but two ligands $L_{1}$ and $L_{2}$ bind a single receptor $R$. This results in two different complexes, $R L_{1}$ and $R L_{2}$, with different internalisation rates. For instance, one of the ligands is produced endogenously, and the other is a drug which is supplied in order to inhibit or stimulate the pharmacological effect caused by the endogenous ligand (cf. Benson et al. 22,23).

Recently, several authors have derived different drugdrug interaction models associated with TMDD with a

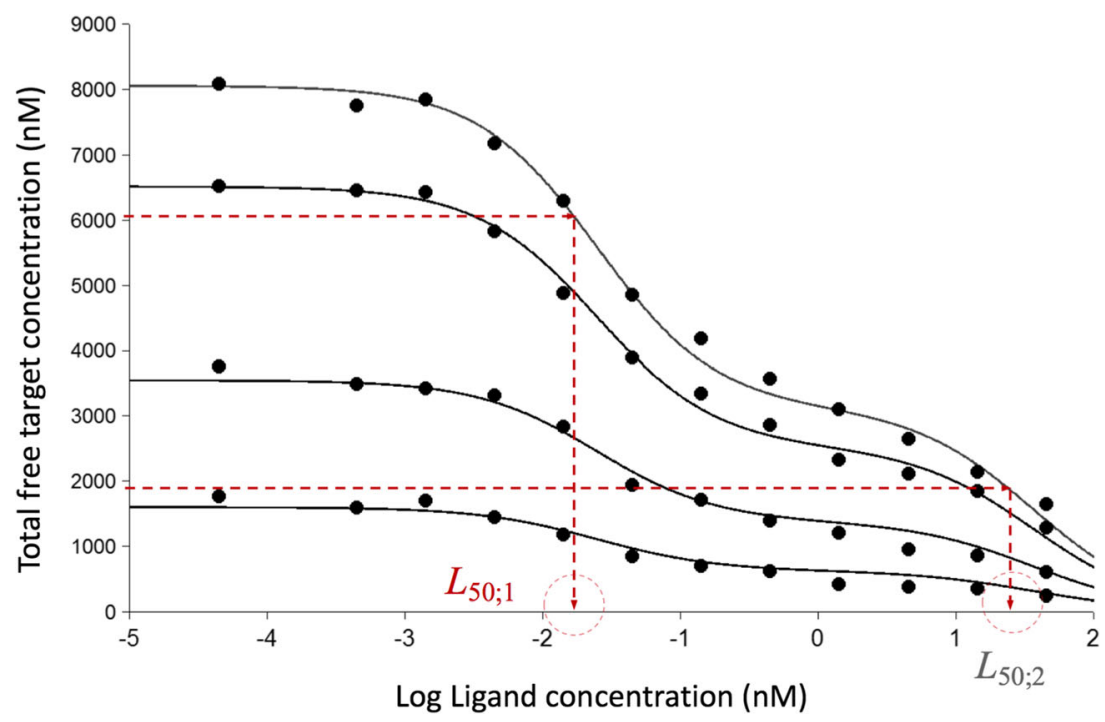

Fig. 5. Total free target level $\boldsymbol{R}_{\text {tot;free }}=\boldsymbol{R}_{\mathbf{1}}+\boldsymbol{R}_{\mathbf{2}}$ and model predicted graphs (solid lines) of

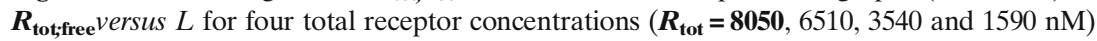
and $\boldsymbol{R}_{\mathbf{0 , 1}}=\boldsymbol{R}_{\text {tot }} \cdot \boldsymbol{F}$ and $\boldsymbol{R}_{\mathbf{0 , 2}}=\boldsymbol{R}_{\text {tot }} \cdot(\mathbf{1}-\boldsymbol{F})$ with $\boldsymbol{F}=\mathbf{0 . 6}$ Note the wide discrepancy between the two in vivo potencies $\boldsymbol{L}_{\mathbf{5 0 ; 1}}$ (denoted in the figure by $\left.\boldsymbol{I}_{\mathbf{5 0 ; 1}}\right)\left(0.025 \mathrm{nM}\right.$ ) and $\boldsymbol{L}_{\mathbf{5 0 ; 2}}$ (denoted in the figure by $\left.\boldsymbol{I}_{\mathbf{5 0 ; 2}}\right)(37 \mathrm{nM})$. The high affinity drug is the target for therapeutic effect, and the low affinity drug is responsible for an adverse effect (cf. Gabrielsson and Weiner, PD2, page 729 21) 
different focus and often directed towards the situation when a constant target level prevails (cf. Koch et al. $(24,25)$ and Gibiansky et al. (26)). In order to describe open, in vivo, processes, it is necessary to include target turnover, internalisation and drug clearance. This is done in the model shown in Fig. 6 in which two ligands, distinguished by subscripts $i=1$ and 2, are supplied by constant-rate infusions $I n_{i}$ to the central compartment, each having its own volume of distribution $V_{c i}$, nonspecific clearance $C l_{\left(L_{i}\right)}$, binding and dissociation rate $k_{\mathrm{on} ; i}$ and $k_{\mathrm{off} ;}$, and its own internalisation rate $k_{e\left(R L_{i}\right)}$.

Mathematically, the model shown in Fig. 6 can be described by the following system of differential equations for the two ligands, $L_{1}$ and $L_{2}$, the target $R$ and the two ligand-target complexes $R L_{1}$ and $R L_{2}$ (see also (12)):

$$
\left\{\begin{aligned}
\frac{d L_{1}}{d t}= & k_{\mathrm{infus} ; 1}-k_{e\left(L_{1}\right)} L-k_{\mathrm{on} ; 1} L_{1} \cdot R+k_{\mathrm{off} ; 1} R L_{1} \\
\frac{d L_{2}}{d t}= & k_{\mathrm{infus} ; 2}-k_{e\left(L_{2}\right)} L-k_{\mathrm{on} ; 2} L_{2} \cdot R+k_{\mathrm{off} ; 2} R L_{2} \\
\frac{d R}{d t}= & k_{\mathrm{syn}}-k_{\mathrm{deg}} R-k_{\mathrm{on} ; 1} L_{1} \cdot R+k_{\mathrm{off} ; 1} R L_{1} \\
& -k_{\mathrm{on} ; 2} L_{2} \cdot R+k_{\mathrm{off} ; 2} R L_{2} \\
\frac{d R L_{1}}{d t}= & k_{\mathrm{on} ; 1} L_{1} \cdot R-\left(k_{\mathrm{off} ; 1}+k_{e\left(R L_{1}\right)}\right) R L_{1} \\
\frac{d R L_{2}}{d t}= & k_{\mathrm{on} ; 2} L_{2} \cdot R-\left(k_{\mathrm{off} ; 2}+k_{e\left(R L_{2}\right)}\right) R L_{2}
\end{aligned}\right.
$$

where

$$
k_{\text {infus }, i}=\frac{I n_{i}}{V_{c}} \quad \text { and } \quad k_{e\left(L_{i}\right)}=\frac{C l_{\left(L_{i}\right)}}{V_{c}}, \quad(i=1,2)
$$

Each of the ligands is present in free form $\left(L_{i}\right)$ and in bound form $\left(R L_{i}\right)(i=1,2)$. For the total amount of the two ligands, we then find two balance equations, one for $L_{1}$ and one for $L_{2}$ :

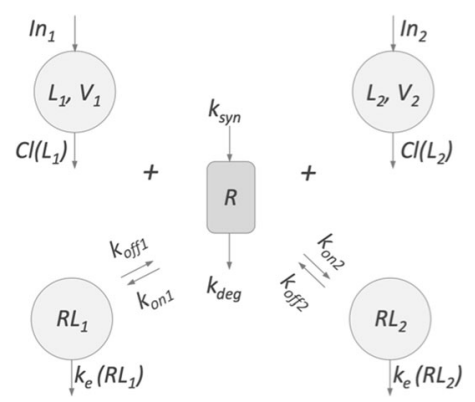

Fig. 6. Schematic description of the competitive-interaction model in which a single target $R$ binds two ligands $\boldsymbol{L}_{\mathbf{1}}$ and $\boldsymbol{L}_{\mathbf{2}}$, forming two complexes denoted by, respectively, $\boldsymbol{R} \boldsymbol{L}_{\mathbf{1}}$ and $\boldsymbol{R} \boldsymbol{L}_{\mathbf{2}}$ $\left\{\begin{array}{l}\frac{d}{d t}\left(L_{1}+R L_{1}\right)=k_{\text {infus } ; 1}-k_{e\left(L_{1}\right)} L_{1}-k_{e\left(R L_{1}\right)} R L_{1} \\ \frac{d}{d t}\left(L_{2}+R L_{2}\right)=k_{\text {infus } ; 2}-k_{e\left(L_{2}\right)} L_{2}-k_{e\left(R L_{2}\right)} R L_{2}\end{array}\right.$

The receptor is present in free form $(R)$ and in bound form $\left(R L_{i}\right)$. Adding the last three equations of the system (Eq. (13)), we obtain the following balance equation for the receptor:

$$
\begin{aligned}
& \frac{d}{d t}\left(R+R L_{1}+R L_{2}\right) \\
& \quad=k_{\mathrm{syn}}-k_{\mathrm{deg}} R-k_{e\left(R L_{1}\right)} R L_{1}-k_{e\left(R L_{2}\right)} R L_{2}
\end{aligned}
$$

These balance equations will be useful for analysing steady-state concentrations, when the left-hand sides vanish and we obtain three algebraic equations.

\section{Steady States}

We deduce from Eq. (16) that the steady-state concentrations $R, R L_{1}$ and $R L_{2}$ are related by the equation

$k_{\mathrm{syn}}-k_{\mathrm{deg}} R-k_{e\left(R L_{1}\right)} R L_{1}-k_{e\left(R L_{2}\right)} R L_{2}=0$

This allows us to express $R$ in terms of the concentrations of the two complexes, $R L_{1}$ and $R L_{2}$ :

$R=\frac{1}{k_{d e g}}\left\{k_{\mathrm{syn}}-k_{e\left(R L_{1}\right)} R L_{1}-k_{e\left(R L_{2}\right)} R L_{2}\right\}$

or

$R=\frac{1}{k_{d e g}}\left(k_{\mathrm{syn}}-X_{1}-X_{2}\right)$.

when we use the short-hand notation

$X_{1}=k_{e\left(R L_{1}\right)} R L_{1} \quad$ and $\quad X_{2}=k_{e\left(R L_{2}\right)} R L_{2}$

We substitute the expression for $R$ in Eq. (19) into the righthand side of each of the last two equations of Eq. (13) to obtain:

$$
\left\{\begin{array}{l}
L_{1} \cdot \frac{1}{k_{d e g}}\left(k_{\mathrm{syn}}-X_{1}-X_{2}\right)=K_{m ; 1} \frac{X_{1}}{k_{e\left(R L_{1}\right)}} \\
L_{2} \cdot \frac{1}{k_{d e g}}\left(k_{\mathrm{syn}}-X_{1}-X_{2}\right)=K_{m ; 2} \frac{X_{2}}{k_{e\left(R L_{2}\right)}}
\end{array}\right.
$$

where

$K_{m, i}=\frac{k_{o f f ; i}+k_{e\left(R L_{i}\right)}}{k_{\text {on } ; i}}$ 
This is an algebraic system of two equations with two unknowns, $X_{1}$ and $X_{2}$, which can be solved. Translating these solutions back to the original variables, we obtain the following expressions for $R L_{1}$ and $R L_{2}$ :

$\left\{\begin{array}{l}R L_{1}=R_{1}^{*} \frac{L_{1}}{L_{1}+\theta \cdot L_{2}+L_{50 ; 1}} \\ R L_{2}=R_{2}^{*} \frac{L_{2}}{L_{2}+\theta^{-1} \cdot L_{1}+L_{50 ; 2}}\end{array}\right.$

where $L_{50 ; 1}$ and $L_{50 ; 2}$ are given by

$L_{50 ; i}=\frac{k_{d e g}}{k_{e\left(R L_{i}\right)}} \cdot K_{m ; i}, \quad R_{i}^{*}=\frac{k_{d e g}}{k_{e\left(R L_{i}\right)}} \quad$ and $\quad \theta=\frac{L_{50 ; 1}}{L_{50 ; 2}}$

for $i=1$ and $i=2$.

The expressions for the complexes $R L_{1}$ and $R L_{2}$ can be used in Eq. (18) to derive an expression for $R$ in terms of the two ligand concentrations:

$R=R_{0}\left(1-\frac{L_{1}}{L_{1}+\theta \cdot L_{2}+L_{50 ; 1}}-\frac{L_{2}}{L_{2}+\theta^{-1} \cdot L_{1}+L_{50 ; 2}}\right)$

where $\theta=L_{50 ; 1} / L_{50 ; 2}$. Thus, the impact of the two ligand combined is seen to be additive.

Details of the derivations of the equations above are given in Appendix 1.2.

In the expressions for $R L_{1}$ in Eq. (22), one can interpret the term $\left(\theta \cdot L_{2}+L_{50 ; 1}\right)$ in the numerator as a shift of potency $L_{50 ; 1}$, and similarly in the expression for $R L_{2}$, the term $\left(\theta^{-1}\right.$. $L_{1}+L_{50 ; 2}$ ) can be viewed as a shift of potency $L_{50 ; 2}$. Thus, the modifications of the potencies $L_{50 ; 1}$ and $L_{50 ; 2}$ (equivalent to $E C_{50 ; 1}$ and $E C_{50 ; 2}$ ) depend on the ligand concentrations in the following manner:

$E C_{50 ; 1} \nearrow \quad$ when $L_{2} \nearrow$

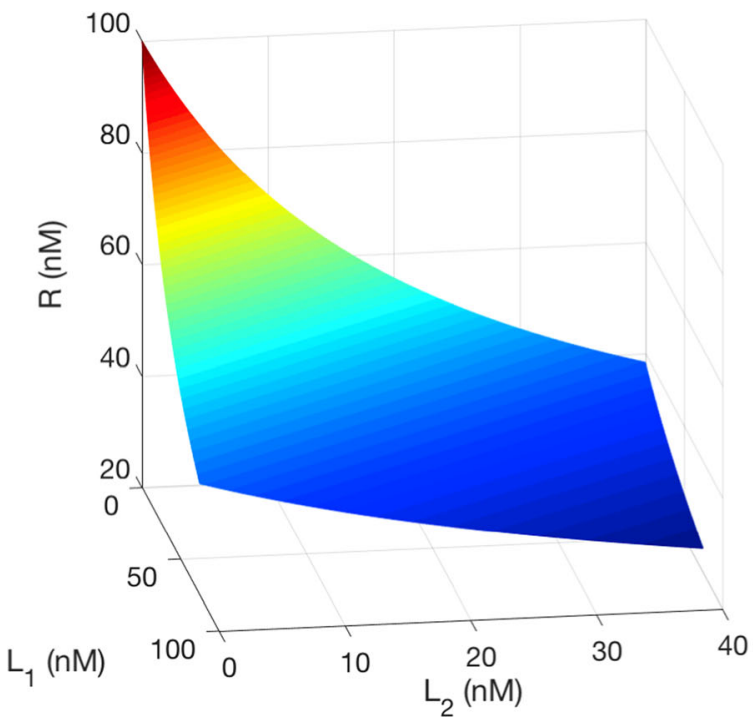

i.e. $E C_{50 ;}$ increases when $L_{2}$ increases. Similarly, $E C_{50 ; 2}$ increases when $L_{1}$ increases.

Note that by Eq. (22), when $L_{2}$ is arbitrary but fixed, then

$R L_{1}\left(L_{1}, L_{2}\right) \rightarrow\left\{\begin{array}{lll}0 & \text { as } & L_{1} \rightarrow 0 \\ R L_{1} \rightarrow R_{1}^{*} & \text { as } & L_{1} \rightarrow \infty\end{array}\right.$

In the context of an endogenous ligand $\left(L_{1}\right)$ and a drug $\left(L_{2}\right)$ which is administered to reduce the effect of the endogenous ligand, Eq. (22) is of practical value. Assuming that receptor occupancy $R L_{1}$ is a measure for the effect of $L_{1}$, it tells by how much the effect of $L_{1}$ is reduced by a given concentration of $L_{2}$.

Finally, we observe that

$\left\{\begin{array}{llll}R L_{1}\left(L_{1}, L_{2}\right) & \rightarrow R_{1}^{*} \frac{L_{1}}{L_{1}+L_{50 ; 1}} \quad \text { as } & L_{2} \rightarrow 0 \\ R L_{2}\left(L_{1}, L_{2}\right) & \rightarrow R_{2}^{*} \frac{L_{2}}{L_{2}+L_{50 ; 2}} \quad \text { as } & L_{1} \rightarrow 0\end{array}\right.$

These limits are consistent with the expression in Eq. (2) for a single receptor shown in "INTRODUCTION". Plainly, $R L_{1}\left(L_{1}, L_{2}\right)=0$ when $L_{1}=0$ and $R L_{2}\left(L_{1}, L_{2}\right)=0$ when $L_{2}=0$.

It is illustrative to view the two complexes and the total free drug concentration as they depend on both ligand concentrations: $L_{1}$ and $L_{2}$. This is done in Fig. 7 where $3 \mathrm{D}$ graph of $R$ versus $L_{1}$ and $L_{2}$ is shown as well as the corresponding Heat map. In both graphs, $R_{0}=100, L_{50 ; 1}=$ 50 and $L_{50 ; 2}=25$ are taken so that $\theta=2$. As we see

$$
\left\{\begin{array}{llll}
R\left(L_{1}, L_{2}\right) & \rightarrow R_{0}=100 & \text { as } & \left(L_{1}, L_{2}\right) \rightarrow(0,0) \\
R\left(L_{1}, L_{2}\right) & \rightarrow R L_{1}\left(0, L_{2}\right)=0 & \text { as } & L_{1} \rightarrow 0
\end{array} .\right.
$$

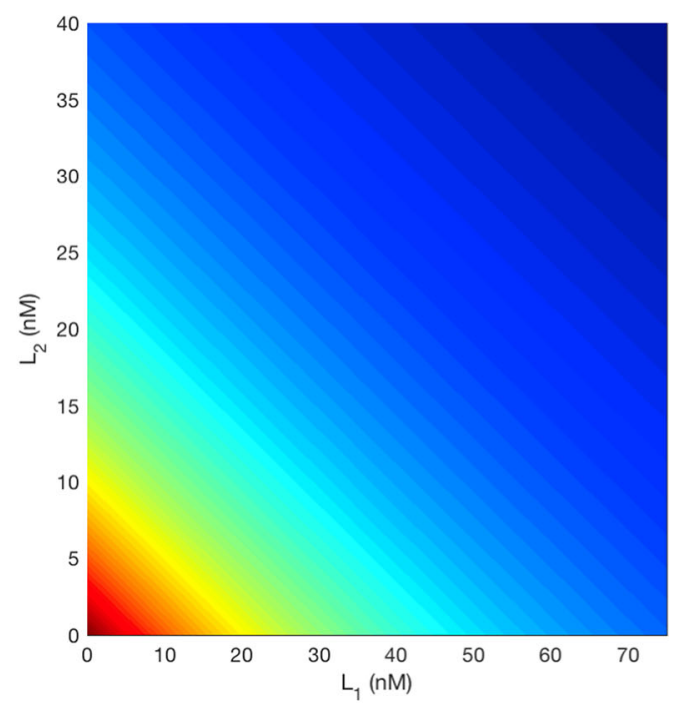

Fig. 7. Graphs of $R$ versus $\boldsymbol{L}_{\mathbf{1}}$ and $\boldsymbol{L}_{\mathbf{2}}$ according to Eq. (24). Here, $\boldsymbol{R}_{\mathbf{0}}=\mathbf{1 0 0}, \boldsymbol{L}_{\mathbf{5 0 ; 1}}=\mathbf{5 0}$ and $\boldsymbol{L}_{\mathbf{5 0 ; 2}}=\mathbf{2 5}$ so that $\boldsymbol{\theta}=\mathbf{2}$. Note that the level curves are straight lines with slope $\boldsymbol{L}_{\mathbf{1}} / \boldsymbol{L}_{\mathbf{2}}=\mathbf{- 2}$ 
These limits are in agreement with the Eq. (22) for $R L_{1}$ and Eq. (25) for $R$.

\section{Discussion and Conclusion}

Equations (22), (23) and (24) summarise what is needed to apply and explain target $R$, ligand-target complex $R L_{i}$ and ligand $L_{i}$ interactions when two ligands interact with one centrally located target. Equation (23) clearly demonstrates that in vivo potency is a conglomerate of target turnover, complex kinetics and ligand-target binding properties.

\section{TARGET IN THE PERIPHERAL COMPARTMENT}

\section{Background}

When ligand and target are located in the central compartment of the TMDD model, the steady-state relations of ligand, target and ligand-target complex have been derived in Gabrielsson and Peletier (1) and briefly summarised in the "Introduction" (cf Eqs. (2) and (3)). In this section, we generalise this situation to when ligand is supplied to the central compartment, but target is located in the peripheral compartment so that ligand has to be cleared from the central compartment into the peripheral compartment before it can bind the target.

We assume active transport between the two compartments, as may be caused by blood flow or transporters, and denote clearance from the central compartment by $C l_{d \alpha}$ and from the peripheral compartment by $C l_{d \beta}$. These two processes allow concentration differences to build up across the membrane separating the two compartments..

The objective is here to derive expressions for the concentration of free receptor $R$ and ligand-receptor complex $R L_{p}$ in the peripheral compartment and the ligand concentration $L_{c}$ in the central compartment. study.
The system (1) now becomes

$$
\left\{\begin{aligned}
\frac{d L_{c}}{d t} & =\frac{I n_{i}}{V_{c}}+\frac{1}{V_{c}}\left(C l_{d \beta} L_{p}-C l_{d \alpha} L_{c}\right)-\frac{C l_{(L)}}{V_{c}} L_{c} \\
\frac{d L_{p}}{d t} & =\frac{1}{V_{p}}\left(C l_{d \alpha} L_{c}-C l_{d \beta} L_{p}\right)-k_{\mathrm{on}} L_{p} \cdot R+k_{\mathrm{off}} R L_{p} \\
\frac{d R}{d t} & =k_{\mathrm{syn}}-k_{\mathrm{deg}} R-k_{\mathrm{on}} L_{p} \cdot R+k_{\mathrm{off}} R L_{p} \\
\frac{d R L_{p}}{d t} & =k_{\mathrm{on}} L_{p} \cdot R-\left(k_{\mathrm{off}}+k_{e\left(R L_{p}\right)}\right) R L_{p}
\end{aligned}\right.
$$

For convenience, we shall often write

$$
\begin{array}{ll}
k_{\text {infus }}=\frac{I n_{i}}{V_{c}} & k_{e(L)}=\frac{C l_{(L)}}{V_{c}}, \\
k_{\mathrm{cp}}=\frac{C l_{d \alpha}}{V_{c}}, & k_{\mathrm{pc}}=\frac{C l_{d \beta}}{V_{p}} .
\end{array}
$$

The system (28) yields the following balance equations for the target and the ligand:

- $\quad$ For the target, which involves free target $R$ and bound target $R L_{p}$ : By adding the third and fourth equation of Eq. (28), we obtain

$\frac{d}{d t}\left(R+R L_{p}\right)=k_{\mathrm{syn}}-k_{\mathrm{deg}} R-k_{e\left(R L_{p}\right)} R L_{p}$

- $\quad$ For the ligand, which involves $L_{c}, L_{p}$ and $R L_{p}$ : By adding the first equation in Eq. (28) and the sum of the second and the fourth equation multiplied by $\mu=V_{p} / V_{c}$, we obtain

$$
\frac{d}{d t}\left\{L_{c}+\mu\left(L_{p}+R L_{p}\right)\right\}=k_{\text {infus }}-k_{\operatorname{deg}} R-\mu \cdot k_{e\left(R L_{p}\right)} R L_{p}
$$

\section{Steady States}

An expression for the concentration of ligand-target complex in terms of the ligand concentration in the peripheral compartment $L_{p}$ can be derived in a manner which is

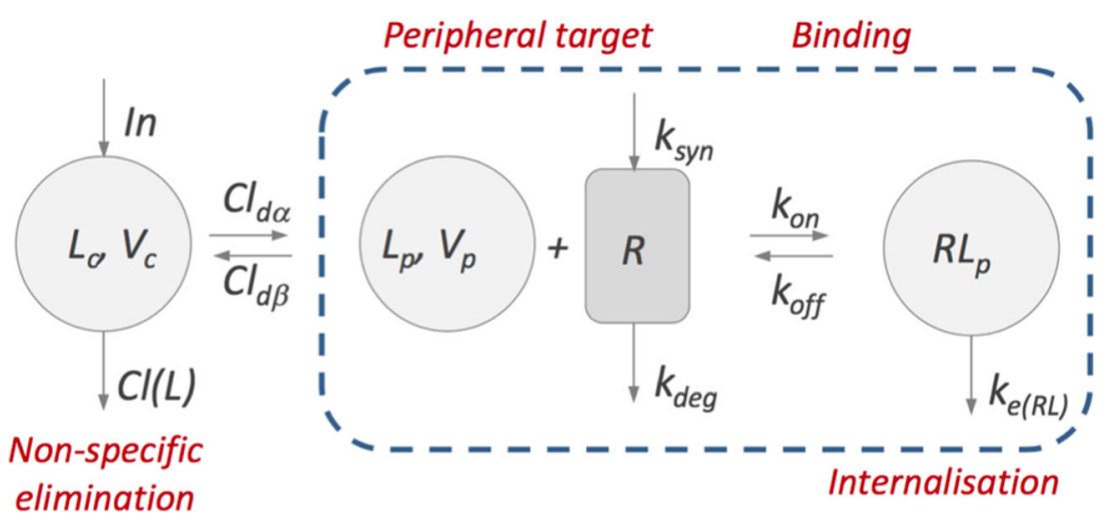

Fig. 8. Schematic description of the model for Target Mediated Drug Disposition involving ligand in the central compartment $\left(\boldsymbol{L}_{\boldsymbol{c}}\right)$ and in the peripheral compartment $\left(\boldsymbol{L}_{\boldsymbol{p}}\right)$ binding a receptor $(R)$ (the target), located in the peripheral compartment yielding ligand-target complexes $\left(\boldsymbol{R} \boldsymbol{L}_{\boldsymbol{p}}\right)$ 
analogous to the one employed before for the onecompartment model and yields the following equation:

$R L_{p}=\frac{k_{s y n} \quad L_{p}}{k_{e\left(R L_{p}\right)} L_{p}+L_{p ; 50}}, \quad$ where $\quad L_{p ; 50}=\frac{k_{d e g}}{k_{e\left(R L_{p}\right)}} \cdot K_{m}$

Note that the expression for $L_{p ; 50}$ is the same as the one defined for $L_{50}$ in Eq. (3).

Next, we replace $L_{p}$ in the expression for $R L_{p}$ by $L_{c}$. By adding the second and the fourth equation of the system (Eq. (28)), we express $L_{p}$ in terms of $L_{c}$ and $R L_{p}$ :

$L_{p}=\frac{1}{k_{p c}}\left\{\mu^{-1} k_{\mathrm{cp}} L_{c}-k_{e\left(R L_{p}\right)} R L_{p}\right\}$.

When we use this equation in Eq. (32) to replace $L_{p}$ by $L_{c}$, we arrive at an expression which only involves $L_{c}$ and $R L_{p}$. Specifically, putting $X=k_{e\left(R L_{p}\right)} R L_{p}$, we obtain

$L_{c}=f(X) \stackrel{\text { def }}{=} \frac{\mu}{k_{c p}}\left(X+\frac{k_{\mathrm{pc}} k_{d e g}}{k_{e\left(R L_{p}\right)}} K_{m} \cdot \frac{X}{k_{s y n}-X}\right)$

Equation (34) provides an expression for $L_{c}$ as a function of $X$, i.e. $L_{c}=f(X)$. It is seen that the function $f(X)$ is monotonically increasing so that it can be inverted to give an expression of $X$ in terms of $L_{c}$ and so yield the desired expression of $R L_{p}$ in terms of $L_{c}$.

In order to invert the function $f(X)$, we multiply Eq. (34) by $\left(k_{\mathrm{syn}}-X\right)$ and so obtain a quadratic equation in $X$ :

$X^{2}-\left(k_{\mathrm{syn}}+a L_{c}+b\right) X+a k_{\mathrm{syn}} L_{c}=0$

in which

$a=\frac{k_{\mathrm{cp}}}{\mu}=k_{p c}$ and $b=\frac{k_{\mathrm{pc}} k_{\mathrm{deg}}}{k_{e\left(R L_{p}\right)}} \cdot k_{m}$

The roots of this equation are

$X_{ \pm}=\frac{1}{2}\left\{\left(k_{\mathrm{syn}}+a L_{c}+b\right) \pm \sqrt{\left(k_{\mathrm{syn}}+a L_{c}+b\right)^{2}-4 a k_{\mathrm{syn}} L_{c}}\right\}$

Obviously, we need the root which vanishes when $L_{c}=0$, i.e. we need $X_{-}$. Therefore

$R L_{p}=\frac{1}{2 k_{e\left(R L_{p}\right)}}\left\{\left(k_{\mathrm{syn}}+a L_{c}+b\right)-\sqrt{\left(k_{\mathrm{syn}}+a L_{c}+b\right)^{2}-4 a k_{\mathrm{syn}} L_{c}}\right\}$

The corresponding expression for target depression in terms of the ligand concentration in plasma $\left(L_{c}\right)$ is found to be given by

$$
R=\frac{k_{s y n}}{2}-\frac{1}{2}\left\{\left(a L_{c}+b\right)-\sqrt{\left(k_{\mathrm{syn}}+a L_{c}+b\right)^{2}-4 a \cdot k_{\mathrm{syn}} L_{c}}\right\}
$$

A more detailed derivation of these expressions for the concentration of ligand-receptor complex in terms of the ligand concentration in plasma can be found in the Appendix 1.1 (A.3 and A.4).

On the basis of the implicit expression (34) of $X$ (i.e. $R L_{p}$ ) in terms of $L_{c}$, it is also possible to define $L_{c}$; 50. Plainly, at $L_{c} ; 50$, we have $X=k_{\text {syn }} / 2$, i.e. $R L_{p}=R^{*} / 2$. When we substitute this value for $X$ into Eq. (34), we obtain the following formula for $L_{c}$; 50 :

$L_{c ; 50}=\frac{\mu}{k_{c p}}\left(\frac{k_{s y n}}{2}+\frac{k_{p c} k_{d e g}}{k_{e\left(R L_{p}\right)}} K_{m}\right)$

or, when we replace the rates $k_{\mathrm{cp}}$ and $k_{\mathrm{pc}}$ by clearances again, we obtain

$L_{c ; 50}=\frac{1}{2} \frac{k_{s y n}}{C l_{d \alpha} / V_{p}}+\frac{C l_{d \beta}}{C l_{d \alpha}} L_{p ; 50}$

where we have used the definition of $L_{p} ; 50$ in Eq. (32).

\section{Passive Transport Between Central and Peripheral Compartment}

If distribution between the two compartments is passive, i.e. $C l_{d \alpha}=C l_{d \beta}=C l_{d}$, then the expression for $L_{c ; 50}$ reduces to $L_{c ; 50}=\frac{1}{2} \frac{k_{s y n}}{C l_{d} / V_{p}}+L_{p ; 50}$

Observation. Equation (40) immediately implies that

$C l_{d \beta}>C l_{d \alpha} \Rightarrow L_{c ; 50}>L_{p ; 50}$

If target-synthesis is small compared to in- and out-flow of ligand between the two compartments, the reverse inequalities are seen to hold as well.

It follows from this expression that $L_{c}$, 50 increases when transport from the central towards the peripheral compartment becomes harder $\left(C l_{d \alpha^{\nearrow}}\right)$ and vice versa, it decreases when it becomes easier $\left(C l_{d \beta} \downarrow\right)$.

If $k_{\text {syn }}$ is small, more specifically, if

$\frac{k_{s y n}}{2} \ll \frac{k_{d e g}}{k_{e\left(R L_{p}\right)}} \frac{C l_{\beta}}{V_{p}} K_{m}$

then, the expression (Eq. (40)) reduced to a particularly simple relation between $L_{c} ; 50$ and $L_{p} ; 50$ : 


$$
L_{c ; 50} \approx \frac{C l_{\beta}}{C l_{\alpha}} L_{p ; 50}
$$

in which the relative impact of the two clearances becomes very transparent.

The expression (Eq. (37)) for $R L_{p}$ in terms of $L_{c}$ is fairly complex and not so easy to grasp. However, it is possible to derive a few properties of the dependence of $R L_{p}$ on $L_{c}$ without going to the details of an explicit computation based on Eq. (37). Below we give a few examples.

1. It follows from Eq. (37) that $0<X<k_{\text {syn. Therefore, }}$ remembering that $X=k_{e\left(R L_{p}\right)} R L_{p}$, it follows that

$R L_{p}<\frac{k_{s y n}}{k_{e\left(R L_{p}\right)}}=R^{*}$

regardless of the ligand concentration $L_{c}$ in the central compartment.

2. It is clear from Eq. (34) that $L_{c}$ is an increasing function of $X$. Therefore, $R L_{p}$ is an increasing function of $L_{c}$ :

$R L_{p}\left(L_{c}\right) \succ R^{*} \quad$ as $\quad L_{c} \rightarrow \infty$

Note that in Eqs. (44) and (45), the active transport between the central and the peripheral compartment (the parameters $\alpha$ and $\beta$ ) do not come into the upper bound and the limit for large $L_{c}$.

In Fig. 9, we show graphs of $R$ and $R L_{p}$ versus $L_{c}$ for three values of the clearance into the peripheral compartment $C l_{d \alpha}(\alpha=0.001, \alpha=1$ and $\alpha=100)$, whilst reverse clearance, from the peripheral compartment into the central compartment is fixed. As predicted by Eq. (40), the potency $L_{c}$;

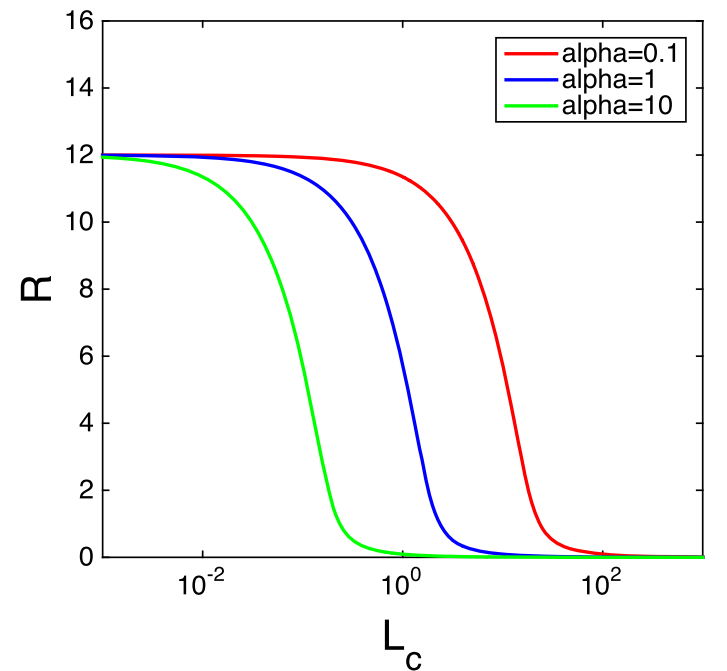

${ }_{50}$ decreases as $\alpha$ increases and hence when $k_{\mathrm{cp}}$ decreases. Of course, this is understandable: When transport to the peripheral compartment becomes easier, drug reaches its target more easily, less of it is required to achieve the same effect and the in vivo potency increases.

Comparing the graphs of the concentration of the ligandtarget complex $R L_{p}$ versus the ligand concentration in the central compartment $L_{c}$ in Figs. 2 and 9, the latter, when target is located peripherally, shows up to be (i) asymmetrical and (ii) to exhibit a shift between the central and peripheral concentrations.

\section{Discussion and Conclusions}

Equations (32), (37), (38) and (39) summarise what is needed to apply and explain target $R$, ligand-target complex $R L_{p}$ and ligand $L\left(L_{c}\right.$ and $\left.L_{p}\right)$ interactions when the target is peripherally located. The effect of the permeability of the membrane between central and peripheral compartment and the volumes of these compartments show up explicitly in the expression for the in vivo potency given in Eq. (39) in combination with target turnover and ligand-target binding properties. This explicit expressions make it possible to give quantitative estimates.

\section{DISCUSSION AND CONCLUSIONS}

\section{In Vivo Potency-the Role of Target Dynamics}

The new concept of potency discussed in this paper departs from the previous one, based on the assumption that the actual expression level of target rather than its turnover rate will determine the potency. Thus, looking at the data of two individuals with the same target expression level (concentration), one would assume that the two individuals would require the same drug exposure. In these papers, we have shown that in fact, this need not be

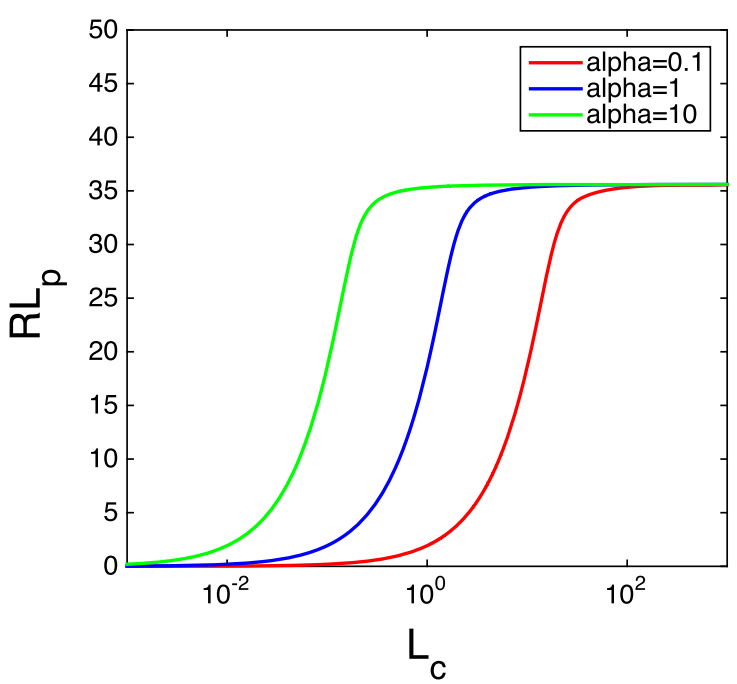

Fig. 9. Sensitivity graphs of $R$ and $\boldsymbol{R} \boldsymbol{L}_{\boldsymbol{p}}$ versus $\boldsymbol{L}_{\boldsymbol{c}}$, on a semi-logarithmic scale, with regard to the clearance rate from the central to the peripheral compartment $\alpha \boldsymbol{C l}_{\boldsymbol{d}}$ where $\boldsymbol{\alpha}=\mathbf{0 . 1 , 1 , 1 0}$ and the clearance rate from peripheral to central compartment $\boldsymbol{\beta} \boldsymbol{C}_{\boldsymbol{d}}$ is fixed $(\boldsymbol{\beta}=\mathbf{1})$. Other parameters are listed in Table I 
true. Instead, according to the definition of $L_{50}$, the subject with the higher target elimination rate $\left(k_{\mathrm{deg}}\right)$ will need more drug compared to a subject with a slow target turnover rate, whilst the subject with the higher internalisation rate $\left(k_{e(R L)}\right)$ will require less drug.

$k_{\mathrm{deg} ; \mathrm{A}}<k_{\mathrm{deg} ; \mathrm{B}} \Rightarrow L_{50 ; \mathrm{A}}<L_{50 ; \mathrm{B}}$

Expressed mathematically, we demonstrated how the potency $L_{50}$ is given in open as opposed to closed systems by the definitions

$$
\left\{\begin{aligned}
L_{50} & =\frac{k_{\text {deg }}}{k_{e(R L)}} \cdot \frac{k_{o f f}+k_{e(R L)}}{k_{o n}} & & \text { Open systems } \\
L_{50} & =\frac{k_{o f f}}{k_{o n}} & & \text { Closed systems }
\end{aligned}\right.
$$

When target baseline levels are the same in two subjects, i.e. $R_{0 ; \mathrm{A}}=R_{0 \text {; } \mathrm{B}}$, but one subject, say $\mathrm{B}$ as in Fig. 10 has a

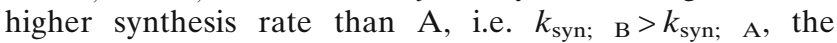
potency of drug in subject $B$ will be numerically higher than in subject $\mathrm{A}$, because $k_{\mathrm{deg}}$; $>k_{\mathrm{deg}}$ A .

\section{Non-symmetric Drug Distribution Between Central and Peripheral Compartment}

We have seen that when target is located in the peripheral rather than the central compartment, the in vivo potency $L_{c}$; 50 will depend in the distributional rates between the two compartments, especially when they are not equal. Indeed, if $C l_{d \alpha}$ denotes clearance out of the central compartment and $C l_{d \beta}$ clearance into the central compartment, then, we have shown that if the synthesis rate of target $k_{\text {syn }}$ is small, the in vivo potency with respect to the ligand concentration in the central compartment $L_{c} ; 50$ and the in vivo potency with respect to the peripheral compartment $L_{p} ; 50$ are related by the simple formula

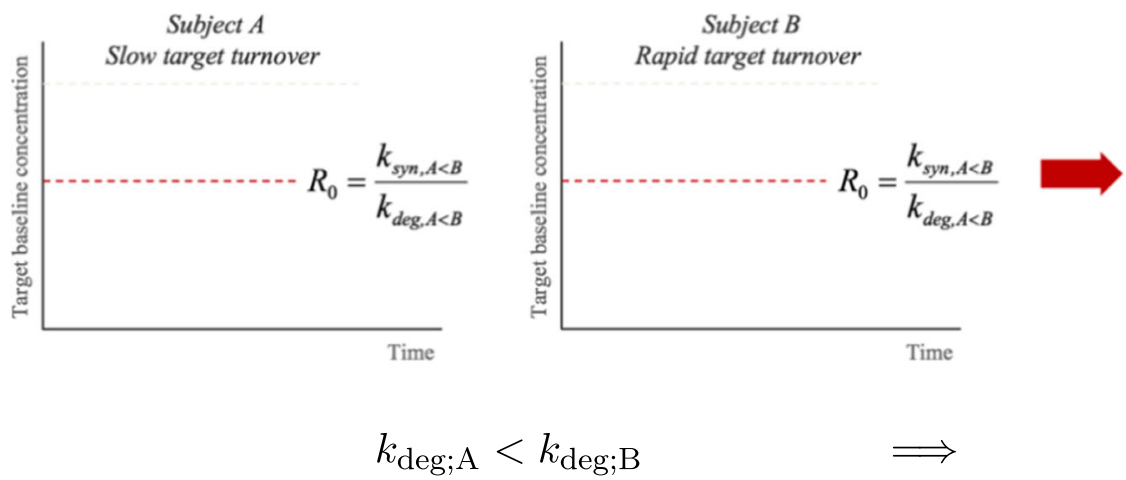

$L_{c ; 50}=\frac{C l_{d \beta}}{C l_{d \alpha}} \cdot L_{p ; 50}$

Thus, if $C l_{d \alpha}>C l_{d \beta}$ relatively easily and high receptor occupancy will be reached for lower ligand concentrations in the central compartment, i.e. $L_{50 c}$ will be relatively small.

On the other hand, when $C l_{d \alpha}<C l_{d \beta}$ ligand has difficulty reaching the target and the potency, $L_{50 ; c}$ will now be larger.

We make two observations about the three graphs in Fig. 10.

1. The graphs appear to be translations of one another with a constant shift.

2. All three graphs have a larger radius of curvature for lower values of $R L_{p}$ and a smaller radius of curvature for higher values of $R L_{p}$.

As regards the first observation, it follows from Eq. (47) that

$\log \left(L_{c ; 50}\right)=\log \left(\frac{C l_{d \beta}}{C l_{d \alpha}}\right)+\log \left(L_{p ; 50}\right)$,

so that the graph shifts by $\log \left(C l_{d \beta} / C l_{d \alpha}\right)$ as we move from one curve to the next in Fig. 10.

\section{Overall Conclusions}

This analysis has focused on the necessity of using an open systems approach for assessment of in vivo pharmacological data.

The major difference between potencies of closed and open systems is that the expression of the latter $\left(L_{50}\right.$ in Eq. (3)) shows that target turnover rate $\left(k_{\mathrm{deg}}\right)$ rather than target concentration $\left(R_{0}\right)$ will determine drug potency. The efficacy (typically denoted $E_{\max } / I_{\max }$ ) of a ligand is, on the other hand, dependent on both target concentration and target turnover rate. When target is located peripherally, the ratio of inter-compartmental distribution $\left(C L_{d \alpha} / C L_{d \beta}\right)$ impacts the potency derived for a centrally located target.

Derived expressions are practically and conceptually applicable when interpreting data translation across individuals, species and studies are done, and also for communication of results to a biological audience.

Fig. 10. Left: Schematic illustrations of the consequences of two subjects with same the baseline target concentration $\left(\boldsymbol{R}_{\mathbf{0}, \boldsymbol{A}}=\boldsymbol{R}_{\mathbf{0}, \boldsymbol{B}}\right)$, but different target turnover rates and losses. Right: Relationships between ligand concentration and normalised target occupancy when target baseline concentration is similar, but fractional turnover rates are different 


\section{APPENDIX 1}

\section{Appendix 1.1. Calculations for two targets}

When ligand can bind two receptors, $R_{1}$ and $R_{2}$, the way the steady-state concentrations of the two complexes $R_{1} L$ and $R_{2} L$ depend on the ligand concentration $L$ can be derived in a manner which very similar to the one used when only one receptor is present. Thus, we deduce from the steady-state equations for $R_{1}$ and $R_{2}$ in the system (6) that

$$
\left\{\begin{array}{l}
R_{1}=\frac{1}{k_{d e g ; 1}}\left(k_{\mathrm{syn}: 1}-X_{1}\right) \\
R_{2}=\frac{1}{k_{d e g ; 2}}\left(k_{\mathrm{syn}: 2}-X_{2}\right)
\end{array}\right.
$$

where we have written $X_{i}=k_{e\left(R_{i} L\right)} R_{i} L(i=1,2)$. Putting the expression for $R_{1}$ into the right-hand side of the equation for $d R_{1} L / d t$ in Eq. (6), and equating it to zero, we obtain

$L\left(k_{\mathrm{syn} ; 1}-X_{1}\right)=K_{m ; 1} \frac{k_{d e g ; 1}}{k_{e\left(R_{1} L\right)}} X_{1}$

where

$K_{m ; 1}=\frac{k_{o f f ; 1}+k_{e\left(R_{1} L\right)}}{k_{o n ; 1}}$

Solving this equation for $X_{1}$ yields

$X_{1}=k_{\mathrm{syn} ; 1} \cdot \frac{L}{L+L_{50 ; 1}} \quad$ where $\quad L_{50 ; 1}=\frac{k_{\text {deg } ; 1}}{k_{e\left(R_{1} L\right)}} \cdot K_{m ; 1}$

from which we obtain for $R_{1} L$ and $R_{1}$ :

$R_{1} L=\frac{k_{s y n}}{k_{e\left(R_{1} L\right)}} \cdot \frac{L}{L+L_{50 ; 1}}$

and

$R_{1}=R_{0 ; 1}-\frac{k_{e\left(R_{1} L\right)}}{k_{d e g ; 1}} R_{1} L=R_{0 ; 1} \frac{L_{50 ; 1}}{L+L_{50 ; 1}}$

the desired expressions given in Eq. (11).

Those for $R_{2} L$ and $R_{2}$ are derived in a similar fashion.

\section{Appendix 1.2. Calculations for two ligands}

When two ligands can bind a single receptor, the dynamics is described by the system (Eq. (13)), which yields the following balance equation for ligand at steady state (cf.
Eq. (16)):

$k_{\mathrm{syn}}-k_{\mathrm{deg}} R-k_{e\left(R L_{1}\right)} R L_{1}-k_{e\left(R L_{2}\right)} R L_{2}=0$

As before, we can express $R$ in terms of $R L_{1}$ and $R L_{2}$ :

$R=\frac{1}{k_{d e g}}\left(k_{\mathrm{syn}}-Y_{1}-Y_{2}\right)$

where we now write $Y_{i}=k_{e\left(R L_{i}\right)} R L_{i}(i=1,2)$.

We substitute this expression for $R$ into the righthand sides of each of the last two equations of the full system (Eq. (13)). Then, we obtain from the one but last equation in Eq. (13):

$L_{1} \cdot \frac{1}{k_{d e g}}\left(k_{\mathrm{syn}}-Y_{1}-Y_{2}\right)=K_{m ; 1} \frac{Y_{1}}{k_{e\left(R L_{1}\right)}}$

and for the last equation of Eq. (13):

$L_{2} \cdot \frac{1}{k_{d e g}}\left(k_{\mathrm{syn}}-Y_{1}-Y_{2}\right)=K_{m ; 2} \frac{Y_{2}}{k_{e\left(R L_{2}\right)}}$.

Equations (A.7) and (A.8) are linear in $Y_{1}$ and $Y_{2}$ and can be solved explicitly. Their solution is

$Y_{1}=k_{\mathrm{syn}} \frac{A_{1}}{1+A_{1}+A_{2}} \quad$ and $\quad Y_{2}=k_{\mathrm{syn}} \frac{A_{2}}{1+A_{1}+A_{2}}$

where

$A_{i}=\frac{L_{i}}{K_{m ; i}} \cdot \frac{k_{e\left(R L_{i}\right)}}{k_{d e g}}, \quad(i=1,2)$

Writing $A_{i}=L_{i} / L_{50 ; i}(i=1,2)$, the expressions for $Y_{1}$ and $Y_{2}$ in (A.10) yield the following relations between $R L_{i}$ and $L_{i}$ :

$$
\begin{aligned}
& R L_{1}=R_{1}^{*} \frac{L_{1}}{L_{1}+\theta \cdot L_{2}+L_{50 ; 1}} \\
& R L_{2}=R_{2}^{*} \frac{L_{2}}{L_{2}+\theta^{-1} \cdot L_{1}+L_{50 ; 2}}
\end{aligned}
$$

where for $i=1,2$,

$L_{50 ; i}=\frac{k_{d e g ; i}}{k_{e\left(R L_{i}\right)}} \cdot K_{m ; i}, \quad R_{i}^{*}=\frac{k_{s y n}}{k_{e\left(R L_{i}\right)}} \quad$ and $\quad \theta=\frac{L_{50 ; 1}}{L_{50 ; 2}}$

Remark. In the expression for $R L_{1}$, one can interpret the term $\left(\theta \cdot L_{2}+L_{50 ; 1}\right)$ as a "potency" related to $L_{1}$, and in the expression for $R L_{2}$, the term $\left(\theta^{-1} \cdot L_{1}+L_{50 ; 2}\right)$ can be viewed as a "potency" related to $L_{2}$. 
Appendix 1.3. Calculations when target is in the peripheral compartment

The four steady-state concentrations $L_{c}, L_{p}, R$ and $R L_{p}$ solve the following set of four algebraic equations:

$$
\begin{cases}k_{\mathrm{infus}}+\mu k_{\mathrm{pc}} L_{p}-k_{\mathrm{cp}} L_{c}-k_{e(L)} L_{c} & =0 \\ \mu^{-1} k_{\mathrm{cp}} L_{c}-k_{\mathrm{pc}} L_{p}-k_{\mathrm{on}} L_{p} \cdot R+k_{\mathrm{off}} R L_{p} & =0 \\ k_{\mathrm{syn}}-k_{\mathrm{deg}} R-k_{\mathrm{on}} L_{p} \cdot R+k_{\mathrm{off}} R L_{p} & =0 \\ k_{\mathrm{on}} L_{p} \cdot R-\left(k_{\mathrm{off}}+k_{e(R L)}\right) R L_{p} & =0\end{cases}
$$

where we recall from "TARGET IN THE PERIPHERAL COMPARTMENT" section that

$k_{\text {infus }}=\frac{I n}{V_{c}} \quad k_{e(L)}=\frac{C l_{(L)}}{V_{c}}$,

$k_{\mathrm{cp}}=\frac{C l_{d \alpha}}{V_{c}}, \quad k_{\mathrm{pc}}=\frac{C l_{d \beta}}{V_{p}}, \quad \mu=\frac{V_{p}}{V_{c}}$.

We now proceed in two steps: (i) We derive a relation between the concentrations of complex and ligand in the peripheral compartment, and then (ii) we derive a comparable relation but between concentrations of the complex in the peripheral compartment $R L_{p}$ and ligand in the central compartment $L_{c}$.

\section{RL $L_{P}$ IN TERMS OF $L_{P}$}

The first equation of Eq. (A.13) yields a relation between the ligand concentrations in the two compartments:

$k_{\text {infus }}+\mu k_{\mathrm{pc}} L_{p}-\left(k_{\mathrm{cp}}+k_{e(L)}\right) L_{c}=0$.

Thus,

$L_{c}=a L_{p}+b k_{\text {infus }}$,

$a=\frac{\mu k_{p c}}{k_{c p}+k_{e(L)}}, \quad b=\frac{1}{k_{c p}+k_{e(L)}}$

We use this expression to eliminate $L_{c}$ from the second equation in Eq. (A.13) and so reduce the system to

$\begin{cases}\left(a k_{\mathrm{cp}}-\mu k_{\mathrm{pc}}\right) L_{p}+b k_{\mathrm{cp}} k_{\text {infus }}+\mu\left(-k_{\mathrm{on}} L_{p} \cdot R+k_{\mathrm{off}} R L_{p}\right) & =0 \\ k_{\mathrm{syn}}-k_{\mathrm{deg}} R-k_{\mathrm{on}} L_{p} \cdot R+k_{\mathrm{off}} R L_{p} & =0 \\ k_{\mathrm{on}} L_{p} \cdot R-\left(k_{\mathrm{off}}+k_{e(R L)}\right) R L_{p} & =0\end{cases}$

Adding the first and $\mu$ times the third equation of Eq. (A.18), we obtain

$\left(a k_{\mathrm{cp}}-\mu k_{\mathrm{pc}}\right) L_{p}+b k_{\mathrm{cp}} k_{\text {infus }}-\mu k_{e(R L)} R L_{p}=0$ and adding the second and the third equation yields

$k_{\mathrm{syn}}=k_{e(R L)} R L_{p}+k_{\mathrm{deg}} R$

We use (A.19) in the fourth equation of Eq. (A.13). Dividing by $k_{\text {on }}$ and multiplying by $k_{\text {deg }}$ yields

$L_{p} \cdot\left(k_{\mathrm{syn}}-k_{e(R L)} R L_{p}\right)=k_{\mathrm{deg}} K_{m} R L_{p}$.

When we now divide by $k_{e(R L)}$ and rearrange the terms, we obtain

$R L_{p}=\frac{k_{s y n} \quad L_{p}}{k_{e(R L)} L_{p}+L_{p ; 50}}, \quad L_{p ; 50}=\frac{k_{d e g}}{k_{e(R L)}} \cdot K_{m}$

Note that this expression for $L_{p} ; 50$ is the same as the one for $L_{50}$ in Eq. (3).

\section{RLP IN TERMS OF $\mathbf{L}_{\mathbf{C}}$}

Whereas in the previous part of Appendix 1.3 we eliminated $L_{c}, R$ and $k_{\text {infus, }}$, we now eliminate $L_{p}, R$ and $k_{\text {infus. }}$ In fact, as before, $k_{\text {infus }}$ is eliminated by means of Eq. (A.16).

(i) Adding the second and the fourth equation of Eq. (A.13), we obtain an expression for $L_{p}$ in therms of $L_{c}$ and $R L_{p}$ :

$L_{p}=\frac{1}{k_{p c}}\left\{\mu^{-1} k_{\mathrm{cp}} L_{c}-k_{e(R L)} R L_{p}\right\}, \quad \mu=\frac{V_{p}}{V_{c}}$

and, as before,

(ii) Adding the third and fourth equation of Eq. (A.13) we obtain, as in Eq. (A.19), for $R$ :

$$
R=\frac{1}{k_{d e g}}\left\{k_{\mathrm{syn}}-k_{e(R L)} R L_{p}\right\}
$$

Finally, we put the expressions for $L_{p}$ and for $R$ into the fourth equation of Eq. (A.13) and obtain, after division by $k_{\text {on, }}$

$\frac{1}{k_{p c}}\left\{\mu^{-1} k_{\mathrm{cp}} L_{c}-X\right\} \times \frac{1}{k_{d e g}}\left\{k_{\mathrm{syn}}-X\right\}=\frac{K_{m}}{k_{e(R L)}} \cdot X$ 
where $X=k_{e(R L)} R L_{p}$. Therefore,

$\mu^{-1} k_{\mathrm{cp}} L_{c}=X+\frac{k_{d e g} k_{p c}}{k_{e(R L)}} K_{m} \cdot \frac{X}{k_{s y n}-X}$

or

$L_{c}=\frac{\mu}{k_{c p}}\left(X+\frac{k_{d e g} k_{p c}}{k_{e(R L)}} K_{m} \cdot \frac{X}{k_{s y n}-X}\right)$

Equation (A.25) provides an expression for $L_{c}$ as a function of $X$, i.e. of $R L_{p}$. Below, we invert this expression and derive a formula for $R L_{p}$ as a function of $L_{c}$ by multiplying Eq. (A.25) by $\left(k_{\mathrm{syn}}-X\right)$ and so obtain the quadratic equation for $X$ :

$X^{2}-\left(k_{\mathrm{syn}}+a L_{c}+b\right) X+a k_{\mathrm{syn}} L_{c}=0$

where

$a=\frac{k_{c p}}{\mu} \quad$ and $\quad b=\frac{k_{d e g} k_{p c}}{k_{e(R L)}} K_{m}$

This is a quadratic equation in $X$ with roots

$X_{ \pm}=\frac{1}{2}\left\{\left(k_{\mathrm{syn}}+a L_{c}+b\right) \pm \sqrt{\left(k_{\mathrm{syn}}+a L_{c}+b\right)^{2}-4 a \cdot k_{\mathrm{syn}} L_{c}}\right\}$

Because, we need the root which vanishes when $L_{c}=0$, i.e. we need $X_{-}$. Thus,

$R L_{p}=\frac{1}{2 k_{e(R L)}}\left\{\left(k_{\mathrm{syn}}+a L_{c}+b\right)-\sqrt{\left(k_{\mathrm{syn}}+a L_{c}+b\right)^{2}-4 a \cdot k_{\mathrm{syn}} L_{c}}\right\}$

Using Eq. (A.22), we deduce the corresponding target depression.

$R=\frac{k_{s y n}}{2}-\frac{1}{2}\left\{\left(a L_{c}+b\right)-\sqrt{\left(k_{\mathrm{syn}}+a L_{c}+b\right)^{2}-4 a \cdot k_{\mathrm{syn}} L_{c}}\right\}$

\section{APPENDIX 2. DATA}

For completeness, we add here the data used in different simulations. Thus, in Fig. 2, we use the data from Peletier and Gabrielsson (10) given in Table I and we use them from Cao and Jusko (18) given in Table II.
Table II. Parameter Values of Cao and Jusko (18)

\begin{tabular}{lllllllll}
\hline$k_{e(L)}$ & $k_{\text {on }}$ & $k_{\text {off }}$ & $k_{\text {syn }}$ & $k_{\text {deg }}$ & $k_{e(R L)}$ & $R_{0}$ & $R_{*}$ & $V$ \\
\hline 0.00038 & 0.1 & 0.001 & 0.1 & 0.01 & 0.03 & 10 & 3.3 & 2.6 \\
$\mathrm{~h}^{-1}$ & $\{(\mathrm{nM}) \mathrm{h}\}^{-1}$ & $\mathrm{~h}^{-1}$ & $(\mathrm{nM}) / \mathrm{h}$ & $\mathrm{h}^{-1}$ & $\mathrm{~h}^{-1}$ & $\mathrm{nM}$ & $\mathrm{nM}$ & $\mathrm{L}$ \\
\hline
\end{tabular}

The data that have been used in studying the competition between two targets for a single ligand in Fig. 4 have been chosen artificially in order to highlight differences binding coefficients, elimination rates and concentrations of the two targets. They are given in Table III.

Table III. Parameter Values for Fig. 4

\begin{tabular}{llllllll}
\hline & $R_{0}$ & $k_{\text {on }}$ & $k_{\text {off }}$ & $k_{\text {syn }}$ & $k_{\text {deg }}$ & $k_{e(R L)}$ & $R^{*}$ \\
\hline Receptor 1 & 100 & 0.753 & 14.6 & 69.4 & 0.694 & 3.93 & 17.6 \\
Receptor 2 & 50 & 1.5 & 14.6 & 35 & 0.694 & 3.93 & 8.9 \\
& $n M$ & $\left\{(n M)\right.$ day $^{-1}$ & day $^{-1}$ & nM day $^{-1}$ & day $^{-1}$ & day $^{-1}$ & nM \\
\hline
\end{tabular}

Open Access This article is distributed under the terms of the Creative Commons Attribution 4.0 International License (http://creativecommons.org/licenses/by/4.0/), which permits unrestricted use, distribution, and reproduction in any medium, provided you give appropriate credit to the original author(s) and the source, provide a link to the Creative Commons license, and indicate if changes were made.

Table I. Parameter Values of Peletier and Gabrielsson (10)

\begin{tabular}{llllllllll}
\hline$k_{\text {on }}$ & $k_{\text {off }}$ & $k_{\text {syn }}$ & $k_{\text {deg }}$ & $C l_{(L)}$ & $C l_{d}$ & $k_{e(R L)}$ & $R_{0}$ & $R_{*}$ & $V_{c}$ \\
\hline 0.091 & 0.001 & 0.1 & 0.0089 & 0.001 & 0.003 & 0.003 & 12 & 36 & 0.05 \\
$(\mathrm{~L} / \mathrm{kg}) / \mathrm{h}$ & $1 / \mathrm{h}$ & $(\mathrm{mg} / \mathrm{L}) / \mathrm{h}$ & $1 / \mathrm{h}$ & $(\mathrm{mg} / \mathrm{L}) / \mathrm{h}$ & $(\mathrm{mg} / \mathrm{L}) / \mathrm{h}$ & $1 / \mathrm{h}$ & $\mathrm{mg} / \mathrm{L}$ & $\mathrm{mg} / \mathrm{L}$ & $\mathrm{L} / \mathrm{kg}$ \\
\hline
\end{tabular}




\section{REFERENCES}

1. Gabrielsson J, Peletier LA. Pharmacokinetic steady-states highlight interesting target-mediated disposition properties. AAPS J. 2017;19(3):772-86.

2. Gabrielsson J, Peletier LA, Hjorth S. In vivo potency revisited-keep the target in sight. Pharmacol Ther. 2017;184:177-88. https://doi.org/10.1016/j.pharmthera.2017.10.011.

3. Wagner JG. Simple model to explain effects of plasma protein binding and tissue binding on calculated volumes of distribution, apparent elimination rate constants and clearances. Eur J Clin Pharmacol. 1976;10:425-32.

4. Sugiyama Y, Hanano M. Receptor-mediated transport of peptide hormones and its importance in the overall hormone disposition in the body. Pharm Res. 1989;6(3):192-202.

5. Levy G. Pharmacologic target mediated drug disposition. Clin Pharmacol Ther. 1994;56:248-52.

6. Michaelis L, Menten ML. Die Kinetik der Invertinwirkung Biochem Z. 1913;49:333-69.

7. Mager DE, Jusko WJ. General pharmacokinetic model for drugs exhibiting target-mediated drug disposition. J Pharmacokinet Phamacodyn. 2001;28(6):507-32.

8. Mager DE, Krzyzanski W. Quasi-equilibrium pharmacokinetic model for drugs exhibiting target-mediated drug disposition. Pharm Res. 2005;22:1589-96.

9. Gibiansky L, Gibiansky E, Kakkar T, Ma P. Approximations of the target-mediated drug disposition model and identifiability of model parameters. J Pharmacokinet Pharmacodyn. 2008;35(5):573-91.

10. Peletier LA, Gabrielsson J. Dynamics of target-mediated drug disposition: characteristic profiles and parameter identification. J Pharmacokinet Pharmacodyn. 2012;39:429-51.

11. Gibiansky L, Gibiansky E. Target-mediated drug disposition model for drugs that bind to more than one target. J Pharmacokinet Pharmacodyn. 2010;37:323-46.

12. Yan X, Chen Y, Krzyzanski W. Methods of solving rapid binding target-mediated drug disposition model for two drugs competing for the same receptor. J Pharmacokinet Pharmacodyn. 2012;39:543-60.

13. Krippendorff BF, Kuester K, Kloft C, Huisinga W. Nonlinear pharmacokinetics of therapeutic proteins resulting from receptor mediated endocytosis. J Pharmacokinet Pharmacodyn. 2009;36:239-60.
14. Black JF, Leff P. Operational models of pharmacological agonism. Proc R Soc Lond B Biol Sci. 1983;220:141-62.

15. Kenakin T. Pharmacologic analysis of drug-receptor interaction. second ed. New York: Raven Press; 1993.

16. Kenakin T. A pharmacology primer: theory, applications, and methods. London: Elsevier Academic Press; 2015.

17. Neubig RR, Spedding M, Kenakin T, Christopoulos A. International Union of Pharmacology Committee on Receptor Nomenclature and Drug Classification. XXXVIII. Update on terms and symbols in quantitative pharmacology. Pharmacol Rev. 2003;55:597-606.

18. Cao Y, Jusko WJ. Incorporating target-mediated drug disposition in a minimal physiologically-based pharmacokinetic model for monoclonal antibodies. J Pharmacokinet Pharmacodyn. 2014;41:375-87.

19. Pang KS, Rodrigues AD, Peter RM, editors. Enzyme- and transporter-based drug-drug interactions: progress and future challenges. New York: Springer; 2010.

20. Pang KS, Huadong S, Chow ECY. Chapter 5. Impact of physiological determinants: flow, binding, transporters and enzymes on organ and total body clearances. In: Pang KS, Rodrigues AD, Peter RM, editors. Enzyme- and transporterbased drug-drug interactions: progress and future challenges. New York: Springer; 2010. p. 107-47. Ê.

21. Gabrielsson J, Weiner D. Pharmacokinetic and pharmacodynamic data analysis, Concept and applications 5th Ed. Apotekarsocieteten, Swedish Pharmaceutical Society, 2016.

22. Benson N, van der Graaf PH, Peletier LA. Cross-membrane signal transduction of receptor tyrosine kinases (RTKs): from systems biology to systems pharmacology. J Math Biol. 2013;66:719-42.

23. Benson N, Graaf PH, van der Peletier LA. Selecting optimal drug-intervention in a pathway involving receptor tyrosine kinases (RTKs). Nonlinear Anal. 2016;137:148-70.

24. Koch G, Jusko WJ, Schropp J. Target-mediated drug disposition with drug-drug interaction, part I: single drug case in alternative formulations. J Pharmacokinet Phamacodyn. 2017;44(1):17-26.

25. Koch G, Jusko WJ, Schropp J. Target mediated drug disposition with drug-drug interaction, part II: competitive and uncompetitive cases. J Pharmacokinet Phamacodyn. 2017;44(1):27-42.

26. Gibiansky L, Gibiansky E. Target-mediated drug disposition model for drugs with two binding sites that bind to a target with one binding site. J Pharmacokinet Phamacodyn. 2016;44(5):463-75. 